\title{
NIELSEN METHODS IN GROUPS WITH A LENGTH FUNCTION
}

\author{
A. H. M. HOARE
}

Many theorems have been proved using cancellation arguments in groups for which a normal form theorem holds. Here we prove a general theorem on groups with an integer valued length function satisfying three of the axioms given by Lyndon ([2]) and show that a large number of cancellation theorems are special cases or immediate corollaries of this theorem.

In section 1 we give definitions and preliminary lemmas. In section 2 we prove the main theorem and two corollaries and in section 3 we give some applications. Further applications will appear in a later paper.

1.

Let $G$ be a group, with identity $e$, which has a normalized integer valued length function, that is a function $x \mapsto|x|$ satisfying

A1'. $|e|=0$,

A2. $|x|=\left|x^{-1}\right|$,

and

A4. $d(x, y)>d(y, z) \quad$ implies $\quad d(x, z)=d(y, z)$,

where

$$
2 d(x, y)=|x|+|y|-\left|x y^{-1}\right| .
$$

[Intuitively $d(x, y)$ is the length of the largest common terminal segment of $x$ and $y$.]

As observed by Lyndon ([2, p. 210]) A4 is equivalent to

$$
d(x, y) \geqq \min \{d(y, z), d(x, z)\}
$$

and to

$$
d(y, z), d(x, z) \geqq m \quad \text { implies } d(x, y) \geqq m .
$$

Received September 21, 1979. In revised form May 27, 1980. 
It can also be shown easily that $\mathrm{A} 1^{\prime}, \mathrm{A} 2$, and $\mathrm{A} 4$ imply

$$
|x| \geqq d(x, y)=d(x, y) \geqq 0 \text {. }
$$

Let $X^{ \pm 1}$ be a subset of $G$. A word in $X^{ \pm 1}$ is a sequence $x_{1} \ldots x_{n}, n \geqq 0$, with $x_{i}$ in $X^{ \pm 1}$. A reduced word is one in which $x_{i+1} \neq x_{i}^{-1}$. A subword is a subsequence, proper or not, of consecutive elements of the sequence. The inverse of $x_{1} \ldots x_{n}$ is $x_{n}^{-1} x_{n-1}^{-1} \ldots x_{1}^{-1}$. We do not distinguish in notation between a word and the group element given by the corresponding product.

Definition. A reduced word $x_{0} x_{1} \ldots x_{n+1}$ is a sink if

$$
\left|x_{0} x_{1} \ldots x_{n}\right|>\left|x_{0} x_{1} \ldots x_{n+1}\right|
$$

and no proper subword or its inverse satisfies the corresponding inequality. A reduced word is sink-free if no subword or its inverse is a sink.

The following extends the Lemma in [1].

LEMMA 1. If every proper subword of the reduced word $w=x_{0} x_{1} \ldots x_{n+1}$ is sink free and if

$$
|w| \leqq \max \left\{\left|x_{0}\right|,\left|x_{1}\right|, \ldots,\left|x_{n+1}\right|\right\}
$$

then

$$
\left|x_{i} x_{i+1} \ldots x_{j}\right|=\max \left\{\left|x_{i}\right|,\left|x_{i+1}\right|, \ldots,{ }^{\prime} ; \mid\right\}
$$

for all proper subwords $x_{i} x_{i+1} \ldots x_{j}$. If strict inequality holds then $\left|x_{0}\right| \geqq\left|x_{1}\right|, \ldots,\left|x_{n}\right| \leqq\left|x_{n+1}\right|$.

Proof. Let $p_{i}=x_{0} x_{1} \ldots x_{i}$ and $q_{i}=x_{i+1} \ldots x_{n+1}$ for $i=0,1, \ldots, n$. Since every proper subword is sink free we have, for $i=1,2, \ldots, n$,

$$
\begin{aligned}
\left|p_{i}\right| & \geqq \max \left\{\left|p_{i-1}\right|,\left|x_{i}\right|\right\}, \\
\left|q_{i-1}\right| & \geqq \max \left\{\left|q_{i}\right|,\left|x_{i}\right|\right\},
\end{aligned}
$$

and by induction

$$
\begin{aligned}
\left|p_{i}\right| & \geqq \max \left\{\left|x_{0}\right|,\left|x_{1}\right|, \ldots,\left|x_{i}\right|\right\}, \\
\left|q_{i-1}\right| & \geqq \max \left\{\left|x_{i}\right|,\left|x_{i+1}\right|, \ldots,\left|x_{n+1}\right|\right\},
\end{aligned}
$$

If

$$
\left|p_{k}\right|+\left|q_{k-1}\right|>\left|p_{k-1}\right|+\left|q_{k}\right|
$$

for some $k=1,2, \ldots, n$ then 


$$
\begin{aligned}
2 d\left(p_{k}, x_{k}\right) & =\left|p_{k}\right|+\left|x_{k}\right|-\left|p_{k-1}\right| \\
& >\left|q_{k}\right|+\left|x_{k}\right|-\left|q_{k-1}\right| \\
& =2 d\left(q_{k}^{-1}, x_{k}\right) .
\end{aligned}
$$

Therefore, by A4, $2 d\left(p_{k}, q_{k}^{-1}\right)=2 d\left(q_{k}^{-1}, x_{k}\right)$ that is, since $w=p_{k} q_{k}$,

$$
\left|p_{k}\right|+\left|q_{k}\right|-|w|=\left|q_{k}\right|+\left|x_{k}\right|-\left|q_{k-1}\right| \text {. }
$$

Suppose that one of the inequalities in (1) is strict for some $k$, then (3) and hence (4) holds for that $k$, and moreover

$$
\begin{aligned}
\left|p_{k}\right|+\left|q_{k-1}\right|-\left|x_{k}\right| & >\max \left\{\left|p_{k-1}\right|,\left|x_{k}\right|\right\}+\max \left\{\left|q_{k}\right|,\left|x_{k}\right|\right\}-\left|x_{k}\right| . \\
& \geqq\left|p_{k-1}\right|,\left|q_{k}\right|,\left|x_{k}\right|,
\end{aligned}
$$

so by (2) and (4)

$$
|w|>\max \left\{\left|x_{0}\right|,\left|x_{1}\right|, \ldots,\left|x_{n+1}\right|\right\}
$$

contradicting the hypothesis. Therefore equalities hold in (1) and hence in (2) for $i=1,2, \ldots, n$. Thus we have proved the first part of the lemma for the proper subwords $p_{j}$ and $q_{i-1}$. However this means that the words $q_{i-1}$ $=x_{i} x_{i+1} \ldots x_{n+1}, i \geqq 1$, also satisfy the hypotheses of the lemma and applying the corresponding equalities in (2) to these words we get

$$
\left|x_{i} x_{i+1} \ldots x_{j}\right|=\max \left\{\left|x_{i}\right|, \ldots,\left|x_{j}\right|\right\}
$$

for $1 \leqq i<j \leqq n$, which together with the result for $p_{j}$ and $q_{i-1}$ proves the first part of the lemma for all $i<j$.

Now by symmetry we may assume $\left|x_{0}\right| \geqq\left|x_{n+1}\right|$. Then strict inequality in the hypothesis gives

$$
\begin{aligned}
|w| & <\max \left\{\left|x_{0}\right|,\left|x_{1}\right|, \ldots,\left|x_{n+1}\right|\right\} \\
& =\max \left\{\left|x_{0}\right|,\left|x_{1}\right|, \ldots,\left|x_{n}\right|\right\} \\
& =\left|p_{n}\right|, \quad \text { by the first part } .
\end{aligned}
$$

Let $k$ be the greatest integer, if any, such that either $\left|p_{k}\right|>\left|p_{k-1}\right|$ or $\left|q_{k-1}\right|$ $>\left|q_{k}\right|$, then (3) and hence (4) holds for that $k$ and moreover

$$
|w|<\left|p_{n}\right|=\left|p_{n-1}\right|=\ldots=\left|p_{k}\right|
$$

SO

$$
0<\left|p_{k}\right|-|w|=\left|x_{k}\right|-\left|q_{k-1}\right| \text { by (4) . }
$$

But $\left|q_{k-1}\right| \geqq\left|x_{k}\right|$ from (1), therefore $\left|p_{i}\right|=\left|p_{i-1}\right|$ and $\left|q_{i-1}\right|=\left|q_{i}\right|$ for all $i$ $=1,2, \ldots, n$. Thus using the first part 


$$
\left|x_{0}\right|=\left|p_{0}\right|=\left|p_{n}\right|=\max \left\{\left|x_{0}\right|,\left|x_{1}\right|, \ldots,\left|x_{n}\right|\right\}
$$

and

$$
\left|x_{n+1}\right|=\left|q_{n}\right|=\left|q_{0}\right|=\max \left\{\left|x_{1}\right|, \ldots,\left|x_{n+1}\right|\right\} .
$$

We now introduce Lyndon's abstract lexicographic ordering on ideals in $G$.

Definition. An ideal is a non-empty subset $\Gamma$ of $G$ such that for any $x$ and $y$ in $\Gamma, z$ is in $\Gamma$ whenever

$$
d(x, z) \geqq d(x, y) .
$$

For any $x$ in $G$ and any integer $i, 0 \leqq i \leqq|x|$, we put

$$
\Gamma_{i}(x)=\{y \in G: 2 d(x, y) \geqq i\},
$$

and we abbreviate $\Gamma_{|x|}(x)$ to $\Gamma(x)$. Each $\Gamma_{i}(x)$ is an ideal and we have a chain

$$
G=\Gamma_{0}(x) \supseteqq \Gamma_{1}(x) \supseteqq \ldots \supseteqq \Gamma_{|x|}(x)=\Gamma(x) .
$$

[Intuitively $\Gamma_{i}(x)$ represents the terminal segment of $x$ of length $i / 2$ and $\Gamma(x)$. the "right half" of $x$ (where $|x|$ may be even or odd).]

Given an arbitrary well-ordering of all the ideals of $G$, we have an induced lexicographic partial well-ordering on the ideals $\Gamma(x)$ defined as follows. If $|x|$ $=\left|x^{\prime}\right|=l$ then put

$$
\Gamma(x)>\Gamma\left(x^{\prime}\right)
$$

whenever, in the chains

$$
\begin{gathered}
G=\Gamma_{0}(x) \supseteqq \Gamma_{1}(x) \supseteqq \ldots \supseteqq \Gamma_{l}(x)=\Gamma(x) \\
G=\Gamma_{0}\left(x^{\prime}\right) \supseteqq \Gamma_{1}\left(x^{\prime}\right) \supseteqq \ldots \supseteqq \Gamma_{l}\left(x^{\prime}\right)=\Gamma\left(x^{\prime}\right),
\end{gathered}
$$

$\Gamma_{i}(x)$ is greater than $\Gamma_{i}\left(x^{\prime}\right)$ (in the given well-order of all the ideals of $G$ ) for the first $i$ for which they are not equal.

REMARK. $\Gamma(x)=\Gamma\left(x^{\prime}\right)$ if and only if

$$
2 d\left(x, x^{\prime}\right) \geqq|x|=\left|x^{\prime}\right| .
$$

Moreover if $2 d\left(x, x^{\prime}\right)<|x|=\left|x^{\prime}\right|$ then we must have

$$
\Gamma(x)<\Gamma\left(x^{\prime}\right) \quad \text { or } \quad \Gamma(x)>\Gamma\left(x^{\prime}\right) .
$$

Suppose that $|x| \geqq|y|$ and $2 d(x, y) \geqq|y|$. If $r \leqq y$ then by A4

$$
2 d(x, z) \geqq r \quad \text { if and only if } \quad 2 d(y, z) \geqq r .
$$


That is

$$
\Gamma_{r}(x)=\Gamma_{r}(y) \quad \text { for all } r \leqq|y| .
$$

Thus we have the following.

Lexicographic Property. If $|x|=\left|x^{\prime}\right| \geqq|y|=\left|y^{\prime}\right|$ and if

$$
2 d(x, y) \geqq|y| \quad \text { and } \quad 2 d\left(x^{\prime}, y^{\prime}\right) \geqq\left|y^{\prime}\right|,
$$

then $\Gamma(x)<\Gamma\left(x^{\prime}\right)$ whenever $\Gamma(y)<\Gamma\left(y^{\prime}\right)$.

[Intuitively this says that if the right halves of $y$ and $y^{\prime}$ are segments of the right halves of $x$ and $x^{\prime}$ respectively, then the right half of $x$ is before the right half of $x^{\prime}$ whenever the right half of $y$ is before the right half of $y^{\prime}$.]

We now use this lexicographic partial well-order to define a partial wellorder on the elements of $G$ as follows.

Put $x<y$ if

(i) $|x|<|y|, \quad$ or

(ii) $|x|=|y| \quad$ and $\quad\left\{\Gamma(x), \Gamma\left(x^{-1}\right)\right\}<\left\{\Gamma(y), \Gamma\left(y^{-1}\right)\right\}$

where the partial order of pairs is defined by

$$
\left\{\Gamma(x), \Gamma\left(x^{-1}\right)\right\}<\left\{\Gamma(y), \Gamma\left(y^{-1}\right)\right\}
$$

if $\Gamma\left(x^{\varepsilon}\right) \leqq \Gamma\left(y^{\eta}\right)$ and $\Gamma\left(x^{-\varepsilon}\right)<\Gamma\left(y^{-\eta}\right)$ for some $\varepsilon, \eta= \pm 1$.

LeMmA 2. If $|x y|=|x| \geqq|y|$ and $\Gamma\left(y^{-1}\right)>\Gamma(y)$ then $x>x y$ and $x \neq y^{ \pm 1}$.

Proof.

$$
2 d\left(x, y^{-1}\right)=|x|+|y|-|x y|=|y|
$$

and

$$
2 d(x y, y)=|x y|+|y|-|x|=|y| .
$$

Therfore by the lexicographic property, $\Gamma\left(y^{-1}\right)>\Gamma(y)$ implies $\Gamma(x)>\Gamma(x y)$. Moreover

$$
2 d\left(x^{-1},(x y)^{-1}\right)=|x|+|x y|-|y| \geqq|x y|=|x|
$$

and so by the Remark above

$$
\Gamma\left(x^{-1}\right)=\Gamma\left((x y)^{-1}\right) .
$$

Thus by condition (ii) of the definition we have $x>x y$.

If $x=y^{-1}$, then $|x y|=0=|x|=|y|$ and $\Gamma(y)=\Gamma_{0}(y)=G=\Gamma\left(y^{-1}\right)$ contradicting 
the hypotheses. If $x=y$, then $|x|=|y|$ and from (5) and the Remark above, $\Gamma\left(y^{-1}\right)=\Gamma(x)$. So $\Gamma\left(y^{-1}\right)=\Gamma(y)$ again contradicting the hypotheses.

2.

For a group with length function we now define a subset, denoted $M$, which plays a central role in what follows.

DEFINITION.

$$
M=\left\{x y \in G: d\left(x, y^{-1}\right)+d\left(y, x^{-1}\right)>|x|=|y|\right\}
$$

[Lyndon ([2, p. 213-214]) showed that a free product with the usual length function satisfies his axiom A5, that is $M=\{e\}$. More generally if $G$ is a free product of $G_{1}$ and $G_{2}$ with amalgamated subgroup $A$, endowed with the usual length function, then $M$ consists of all conjugates of $A$ in $G$ (see section 3).]

If $X$ is a subset of $G$ and $w=x_{0} x_{1} \ldots x_{n+1}$ is a word in $X^{ \pm 1}$ we define a Nielsen transformation of $X$ attached to $w$ to be a replacement of an element of $X$ occurring in $w$, say $x_{k}$, by $x_{i} x_{i+1} \ldots x_{j}$, where $0 \leqq i \leqq k \leqq j \leqq n+1$ and $x_{k}^{ \pm 1}$ $\neq x_{i}, \ldots, x_{k-1}, x_{k+1}, \ldots, x_{j}$, leaving all other elements of $X$ fixed. We denote this by

$$
x_{k} \mapsto x_{i} x_{i+1} \ldots x_{j}
$$

Clearly the resulting set generates the same subgroup as $X$. If $x_{k}>x_{i} x_{i+1} \ldots x_{j}$ then we say that the Nielsen transformation reduces $x_{k}$.

THEOREM. Let $X$ be a subset of $G$ which is minimal under Nielsen transformations attached to a word $w=x_{0} x_{1} \ldots x_{n+1}, x_{i} \in X^{ \pm 1}$. Suppose $w$ satisfies the hypotheses of Lemma 1 , and is not $x x^{-1}$, for $x \in X^{ \pm 1}$, then

(i) $\left|x_{i-1}\right|>\left|x_{i}\right|=\ldots=\left|x_{j}\right|<\left|x_{j+1}\right|$ implies

$$
\Gamma\left(x_{i}^{-1}\right)=\Gamma\left(x_{i}\right)=\ldots=\Gamma\left(x_{j}^{-1}\right)=\Gamma\left(x_{j}\right) .
$$

(ii) $\left|x_{0}\right|=\left|x_{1}\right|=\ldots=\left|x_{n+1}\right|$ implies

$$
\Gamma\left(x_{0}^{-1}\right) \geqq \Gamma\left(x_{0}\right)=\Gamma\left(x_{1}^{-1}\right)=\Gamma\left(x_{1}\right)=\ldots=\Gamma\left(x_{n+1}^{-1}\right) \leqq \Gamma\left(x_{n+1}\right),
$$

and, if $|w|<\left|x_{0}\right|$, then $w \in M$ and either $x_{0}=x_{n+1}^{-1}$, or both $\Gamma\left(x_{0}^{-1}\right)=\Gamma\left(x_{0}\right)$ and $\Gamma\left(x_{n+1}^{-1}\right)=\Gamma\left(x_{n+1}\right)$.

(iii) $\left|x_{0} x_{1} \ldots x_{n+1}\right|<\max \left\{\left|x_{0}\right|,\left|x_{1}\right|, \ldots,\left|x_{n+1}\right|\right\}$ implies $\left|x_{0}\right|=\left|x_{n+1}\right|, x_{0}=x_{i}^{ \pm 1}$ for some $i=1,2, \ldots, n+1$, and $x_{n+1}=x_{j}^{ \pm 1}$ for some $j=0,1, \ldots, n$. 
(iv) $\left|x_{i-1}\right|>\left|x_{i}\right|,\left|x_{i+1}\right|, \ldots,\left|x_{j}\right|<\left|x_{j+1}\right|$ implies $x_{i} x_{i+1} \ldots x_{j}$ is in $M$, and

$$
2 d\left(x_{i-1}, x_{j+1}\right) \geqq \min \left\{\left|x_{i-1}\right|,\left|x_{j+1}\right|\right\} .
$$

Proof. Let $n=0$ and $|w|<\max \left\{\left|x_{0}\right|,\left|x_{1}\right|\right\}$, then $x_{0}=x_{1}^{ \pm 1}$, for otherwise $x_{k} \mapsto x_{0} x_{1}$ is an attached Nielsen transformation reducing $x_{k}$ for $k=0$ or 1 . By assumption $x_{0} \neq x_{1}^{-1}$, so $x_{0}=x_{1}$ and both (ii) and (iii) hold. Conclusions (i) and (iv) do not apply in this case. It remains to consider the cases $n>0$, and $n=0$ with $w=\max \left\{\left|x_{0}\right|,\left|x_{1}\right|\right\}$.

Suppose $\left|x_{k-1}\right| \geqq\left|x_{k}\right|$ then, using Lemma 1 in the case $n>0$, we have

$$
\left|x_{k-1} x_{k}\right|=\left|x_{k-1}\right| \geqq\left|x_{k}\right| \text {. }
$$

If $\Gamma\left(x_{k}^{-1}\right)>\Gamma\left(x_{k}\right)$, then by Lemma $2, x_{k-1}>x_{k-1} x_{k}$ and $x_{k-1} \neq x_{k}^{ \pm 1}$, so $x_{k-1} \mapsto x_{k-1} x_{k}$ is an attached Nielsen transformation reducing $x_{k-1}$, which contradicts minimality. Thus $\left|x_{k-1}\right| \geqq\left|x_{k}\right|$ implies $\Gamma\left(x_{k}^{-1}\right) \leqq \Gamma\left(x_{k}\right)$. Similarly, if $\left|x_{k}\right| \leqq\left|x_{k+1}\right|$, then $\Gamma\left(x_{k}^{-1}\right) \geqq \Gamma\left(x_{k}\right)$. Moreover if $\left|x_{k-1}\right|=\left|x_{k}\right|$, then we have equality in (6), and hence by the Remark in section $1, \Gamma\left(x_{k-1}\right)=\Gamma\left(x_{k}^{-1}\right)$. These three facts prove (i) and the first part of (ii).

Suppose $|w|<\left|x_{0}\right|$ and $\Gamma\left(x_{0}^{-1}\right)>\Gamma\left(x_{0}\right)=\ldots=\Gamma\left(x_{n+1}^{-1}\right)$ then $x_{0} \neq x_{1}^{ \pm 1}, \ldots$, $x_{n}^{ \pm 1}, x_{n+1}$. If $x_{0} \neq x_{n+1}^{-1}$, then $x_{0} \mapsto x_{0} \ldots x_{n+1}$ is an attached Nielsen transformation which reduces the length of $x_{0}$ contradicting minimality, so $x_{0}=x_{n+1}^{-1}$. Similarly if $\Gamma\left(x_{n+1}^{-1}\right)<\Gamma\left(x_{n+1}\right)$, then $x_{0}=x_{n+1}^{-1}$.

In either case, $\Gamma\left(x_{0}^{-1}\right)=\Gamma\left(x_{n+1}\right)$, and by the Remark in section 1, $2 d\left(x_{0}^{-1}, x_{n+1}\right) \geqq\left|x_{0}\right|$. Put $x=x_{0} x_{1} \ldots x_{n}$ and $y=x_{n+1}$, then $w=x y$ and

$$
2 d\left(x^{-1}, x_{0}^{-1}\right)=|x|+\left|x_{0}\right|-\left|x_{0}^{-1} x\right| \geqq\left|x_{0}\right|,
$$

so by $\mathrm{A} 4,2 d\left(x^{-1}, y\right) \geqq\left|x_{0}\right|$. Moreover

$$
2 d\left(x, y^{-1}\right)=|x|+|y|-|x y|>\left|x_{0}\right|
$$

so $w$ is in $M$. This completes the proof of (ii).

If $\left|x_{0} x_{1} \ldots x_{n+1}\right|<\max \left\{\left|x_{0}\right|,\left|x_{i}\right|, \ldots,\left|x_{n+1}\right|\right\}$, then by Lemma $1\left|x_{0}\right|$ $\geqq\left|x_{1}\right|, \ldots,\left|x_{n}\right| \leqq\left|x_{n+1}\right|$. Suppose $\left|x_{0}\right|>\left|x_{n+1}\right|$, then $x_{0} \mapsto x_{0} x_{1} \ldots x_{n+1}$ is a Nielsen transformation reducing $x_{0}$, so $\left|x_{0}\right| \leqq\left|x_{n+1}\right|$. Similarly $\left|x_{n+1}\right| \leqq\left|x_{0}\right|$. Therefore $\left|x_{0} x_{1} \ldots x_{n+1}\right|<\left|x_{0}\right|=\left|x_{n+1}\right|$, and because of the minimality neither $x_{0} \mapsto x_{0} x_{1} \ldots x_{n+1}$ nor $x_{n+1} \mapsto x_{0} x_{1} \ldots x_{n+1}$ can be Nielsen transformations. The result (iii) follows.

It remains to prove (iv). Let $x=x_{i-1}, y=x_{i} x_{i+1} \ldots x_{j}$, and $z=x_{j+1}$, then by Lemma $1,|x|=|x y|>|y|<|y z|=|z|$ and $|x y z| \leqq \max \{|x|,|z|\}$. Therefore

$$
2 d\left(x^{-1},(x y)^{-1}\right)=|x|+|x y|-|y|>|x|=|x y| .
$$


By the Remark in section $1, \Gamma\left(x^{-1}\right)=\Gamma\left((x y)^{-1}\right)$, and hence

$$
\Gamma(x) \leqq \Gamma(x y),
$$

otherwise $x>x y$ and $x_{i-1} \mapsto x_{i-1} x_{i} \ldots x_{j}$ is an attached Nielsen transformation reducing $x_{i-1}$. Similarly

$$
\Gamma\left(z^{-1}\right) \leqq \Gamma\left((y z)^{-1}\right) .
$$

We can suppose by symmetry that $|x| \leqq|z|$, so

$$
2 d\left(x y, z^{-1}\right)=|x y|+|z|-|x y z| \geqq|x y|,
$$

and

$$
2 d\left(x,(y z)^{-1}\right)=|x|+|y z|-|x y z| \geqq|x| .
$$

If $\Gamma(x)<\Gamma(x y)$ then by the Lexicographic Property, $\Gamma\left((y z)^{-1}\right)<\Gamma\left(z^{-1}\right)$, contradicting (9). Therefore from (8), $\Gamma(x)=\Gamma(x y)$, that is by the Remark in section 1 ,

$$
2 d(x, x y) \geqq|x|=|x y| .
$$

Thus by A4 using (10)

$$
2 d\left(x, z^{-1}\right) \geqq|x y|=|x|=\min \{|x|,|z|\} .
$$

Moreover from (7), $2 d\left(x^{-1},(x y)^{-1}\right)>|x|=|x y|$, so $y=x^{-1} x y$ is in $M$.

Following Lyndon [2] we put

$$
N=\left\{x \in G: \Gamma(x)=\Gamma\left(x^{-1}\right)\right\}
$$

and if $x$ and $y$ are in $N$ we put $x \sim y$ if $2 d(x, y) \geqq|x|=|y|$. This is easily shown to be an equivalence relation.

Definition. A subset $X$ of $G$ is minimal if there is no Nielsen transformation of $X$ reducing one element of $X$ and leaving the others fixed.

Corollary 1. Let $X$ be minimal and let $H$ be the subgroup generated by $X$. If $H \cap M=\{e\}$ then $X \backslash N$ is a basis for a free subgroup $F$ of $H$, and $H$ is the free product of $F$ and the subgroups generated by equivalent elements of $X \cap N$.

Proof. Suppose $w$ is a reduced word in $X^{ \pm 1}$ which gives the identity in $G$ and which has no proper subword giving the identity. Then either all the letters in $w$ are of length zero and are thus equivalent elements of $N$ or some subword or its inverse say $x_{0} x_{1} \ldots x_{n+1}$ forms a sink. By Lemma 1 and part (iii) of the Theorem 


$$
\left|x_{n+1}\right|=\left|x_{0}\right| \geqq\left|x_{1}\right|, \ldots,\left|x_{n}\right| .
$$

Since $H \cap M=e$, part (iv) of the Theorem cannot occur so

$$
\left|x_{0}\right|=\left|x_{1}\right|=\ldots=\left|x_{n+1}\right|
$$

and, by part (ii) and the definitions of $N$ and $\Gamma(x), x_{0}, x_{1}, \ldots, x_{n+1}$ are equivalent elements of $N$ with $x_{0} x_{1} \ldots x_{n+1}=e$. Thus every relation between elements of $X$ is a consequence of relations between equivalent elements of $X \cap N$. The result follows from the definition of a free product.

Corollary 2. Suppose $G$ can be generated by elements of length zero or one. If $M=\{e\}$ then every minimal set of generators of $G$ has elements of length zero or one only.

Proof. Let $X$ be a minimal set of generators of $G$. Suppose there is some $x$ in $X$ of length greater than one. Consider all words in $X^{ \pm 1}$ which give elements of length zero or one in $G$ and which have no subword giving the identity. Since $G$ is generated by these elements either $x$ is redundant in $X$ or it appears in one of these words. In the first case there is a Nielsen transformation taking $x$ to the identity, contradicting the minimality of $X$, and in the second case we have a sink $x_{0} x_{1} \ldots x_{n+1}$ with no subword giving the identity. By the theorem this is impossible if $M=\{e\}$.

Note that the same proof shows that if $M=\{e\}$ and if $G$ is generated by elements of length less than $r$, then the elements of every minimal set of generators have length less than $r$.

3.

Although it is possible to prove general results about the sets $M$ and $N$ and about the equivalence relation on $N$, we will here apply the Theorem and Corollaries only to special cases.

I. Free products with amalgamation.

Suppose $G$ is a free product of groups $G_{\lambda}$, called the factors, with a common proper amalgamated subgroup $A$. Then for every element $g$ of $G$, not in $A$, there is a smallest integer $l$ such that $g$ is a product of $l$ elements $g_{1} g_{2} \ldots g_{l}$ with successive $g_{i}$ from different factors and not in $A$ (see [4, section 4.2]). Call $g_{1} g_{2} \ldots g_{l}$ a reduced form for $g$ and define $|g|=l$ and $|a|=0$ for all $a \in A$. Then $x \mapsto|x|$ satisfies the axioms of Section 1. If $x_{1} \ldots x_{l}$ and $y_{1} \ldots y_{m}$ are reduced forms for $x$ and $y$ respectively, then $d\left(x, y^{-1}\right)>0$ if and only if $x_{l}$ and $y_{1}$ are from the same factor. Moreover 


$$
x_{l-r+1} \ldots x_{l} y_{1} \ldots y_{r} \in A \quad \text { for } r \leqq d\left(x, y^{-1}\right) .
$$

Let $a$ be in $A$ and let $x$ be an element of $G$ of length $l \geqq 1$. Put $y=a x^{-1}$, then $y x=a, x y=x a x^{-1}$ and

$$
0=|y x| \leqq|x y| \leqq 2 l-1 .
$$

Hence

$$
d\left(x, y^{-1}\right)+d\left(y, x^{-1}\right) \geqq \frac{1}{2}+l>l=|x|=|y|,
$$

so $x_{a x}^{-1}$ and $a$ are in $M$. Thus every conjugate of an element of $A$ is in $M$.

Conversely suppose

$$
d\left(x, y^{-1}\right)+d\left(y, x^{-1}\right)>|x|=|y|=l .
$$

Let $s$ and $t$ be the integer parts of $d\left(x, y^{-1}\right)$ and $d\left(y, x^{-1}\right)$, respectively. Since $2 d\left(x, y^{-1}\right)$ and $2 d\left(y, x^{-1}\right)$ are integers, we have $s+t \geqq l$. Put $r=l-s$, then from (11) we have that $x_{r+1} \ldots x_{l} y_{1} \ldots y_{s}$ and $y_{s+1} \ldots y_{l} x_{1} \ldots x_{r}$ are in $A$, say $a$ and $a^{\prime}$, respectively. Since $x y=x_{1} \ldots x_{r} a a^{\prime}\left(x_{1} \ldots x_{r}\right)^{-1}$, every element of $M$ is a conjugate of an element of $A$.

Similar methods will show that $N$ consists of conjugates of the factors of $G$, each conjugate of each factor being an equivalence class. Thus we have the following.

H. NeumanN's Theorem ([3]). If $G$ is a free product with amalgamated subgroup $A$ and if $H$ is a subgroup which intersects all conjugates of $A$ trivially, then $H$ is a free product of a free group and conjugates of subgroups of the factors of $G$.

If $A$ is the identity, that is $G$ is a free product, then this reduces to the following.

Kuros Subgroup Theorem. Every subgroup of $G$ is a free product of a free group and conjugates of subgroups of the free factors.

If $G$ is a free product with factors $G_{\lambda}$ and if $g\left(G_{\lambda}\right)$ is the minimum number of generators of $G_{\lambda}$, then by Corollary 2 any minimal set $X$ of generators of $G$ consists of elements of the factors $G_{\lambda}$. Moreover, in order to generate $G_{\lambda}, X$ must have at least $g\left(G_{\lambda}\right)$ elements in $G_{\lambda}$.

Grushoo-Neumann Theorem. The cardinality of $X$ is not less than $\sum_{\lambda} g\left(G_{\lambda}\right)$. Moreover if $\sum_{\lambda} g\left(G_{\lambda}\right)$ is finite and $\varphi$ is an epimorphism of a free group $F$ with finite basis $B$ onto $G$, then there is an automorphism $\alpha$ of $F$ such that $\varphi(\alpha(B))$ $\subset \cup_{\lambda} G_{\lambda}$. 
Proof. The first part follows from the immediately preceeding remarks. Since $\varphi(B)$ is finite it can be minimised in the partial well order by a finite number of Nielsen transformation. The result follows since each Nielsen transformation of $\varphi(B)$ can be obtained by an automorphism of $F$.

We now show that Theorem 1 of Zieschang $([6$, p. 11]) is a special case of the Theorem above. Let $G$ be the free product of $G_{\lambda}$ with amalgamated subgroup $A$, and with the length function described above. Let $X$ be a minimal set in $G$. Suppose that $w=x_{0} x_{1} \ldots x_{n+1}, x_{i} \in X^{ \pm 1}$, is a sink, with no subword equal to the identity. Then by Lemma 1 and part (iii) of the Theorem, $\left|x_{0}\right|$ $=\left|x_{n+1}\right| \geqq\left|x_{1}\right|, \ldots,\left|x_{n}\right|$. Let $l=\min \left\{\left|x_{1}\right|, \ldots,\left|x_{n}\right|\right\}$ and let $x_{i} \ldots x_{j}$ be a maximal subword of $w$ such that $\left|x_{i}\right|=\ldots=\left|x_{j}\right|=l$ if $l \geqq 1$, and $\left|x_{i}\right|, \ldots,\left|x_{j}\right| \leqq 1$ if $l=0$. Then exactly one of the following holds:

(a) $l=0, \quad\left|x_{i}\right|=\ldots=\left|x_{j}\right|=0 \quad$ and $\left|x_{i-1}\right|,\left|x_{j+1}\right| \geqq 2$,

(b) $l=0$ and $\left|x_{i}\right|, \ldots,\left|x_{j}\right|$ are all zero or one, with at least one of each; moreover $\left|x_{i-1}\right|,\left|x_{j+1}\right| \geqq 2$, unless $i=0$ and $j=n+1$.

(c) $\left|x_{i}\right|=\ldots=\left|x_{j}\right|=l \geqq 1$; moreover $\left|x_{i-1}\right|,\left|x_{j+1}\right|>l$ unless $i=0$ and $j=n+1$.

If $i \neq 0$ and $j \neq n+1$, let $\xi_{1} \xi_{2} \ldots \xi_{n}$ and $\eta_{1} \ldots \eta_{m}$ be reduced forms for $x_{i-1}$ and $x_{j+1}$, respectively.

In case (a), $x_{i}, \ldots, x_{j}$ are all in $A$ and $m, n \geqq 2$. Moreover by part (iv) of the Theorem, $2 d\left(x_{i-1}, x_{j+1}^{-1}\right) \geqq \min \{m, n\} \geqq 2$, and by Lemma $1,\left|x_{i-1} x_{i} \ldots x_{j} x_{j+1}\right|$ $\leqq \max \{m, n\}$, that is $2 d\left(x_{i-1} \alpha, x_{j+1}^{-1}\right) \geqq \min \{m, n\} \geqq 2$, where $e \neq \alpha=x_{i} \ldots$ $x_{j} \in A$. Therefore, by (11), we have $\xi_{n} \eta_{1}$ and $\left(\xi_{n} \alpha\right) \eta_{1}$ in $A$. Thus $\xi_{n} \alpha \xi_{n}^{-1}$ $=\xi_{n} \alpha \eta_{1} \eta_{1}^{-1} \xi_{n}^{-1} \in A$, where $\xi_{n}$ lies in one of the factors and not in $A$, so (2.3) of Ziechang's theorem ([6, p. 11]) is satisfied.

In case (b), $x_{i}, \ldots, x_{j}$ all lie in the same factor and have length $\leqq 1$. At least one of these has length 1 , i.e. does not lie in $A$. If $i=0$ and $j=n+1$, then $x_{0} x_{1} \ldots x_{n+1}$ is in $A$ and satisfies (2.4) of [6]. If $i \neq 0$ and $j \neq n+1$, then $x_{i} \ldots x_{j}$ $=\alpha$ lies in one of the factors but not in $A$ and, as in case (a), $\xi_{n} \alpha \xi_{n}^{-1}$ lies in $A$; moreover $\xi_{n}$ and $\alpha$ lie in the same factor, and again (2.4) of [6] is satisfied.

In case (c), if $i=0$ and $j=n+1$, then $x_{0}, x_{1}, \ldots, x_{n+1}$ are all of the same length and by part (ii) of the theorem either $x_{0}, x_{1}, \ldots, x_{n+1}$ are equivalent elements of $N$ (i.e. lie in a conjugate of one of the factors) and $x_{0} x_{1} \ldots x_{n+1}$ is in $M$ (i.e. is conjugate to a non-identity element of $A$ ), or $x_{0}=x_{n+1}^{-1}$ and $x_{1}, \ldots, x_{n}$ lie a conjugate of one of the factors and $x_{0} x_{1} \ldots x_{n+1}$, and hence $x_{1} \ldots x_{n}$, is conjugate to a non-identity element of $A$. If $i \neq 0$ snd $j \neq n+1$, then $x_{i}, \ldots, x_{j}$ lie in a conjugate of one of the factors and $x_{i} \ldots x_{j}$ is conjugate to a non-identity element of $A$. Thus in every case (2.4) of [6] is satisfied. We have thus 
completed the proof that Theorem 1 of [6] is a special case of the Theorem above.

II. H.N.N. extensions.

Suppose that $G$ is an H.N.N. group with base $B$ and associated pair $K_{1}, K_{-1}$, then equivalent elements of $N$ lie in the same conjugate of the base and elements of $M$ are conjugates of the associated subgroups. Therefore Corollary 1 gives

H. NeumanN's TheORem ([3]). If $G$ is an H.N.N. group and $H$ is a subgroup of $G$ which intersects all conjugates of the associated pair trivially then $H$ is a free product of a free group and conjugates of subgroups of the base of $G$.

If $X$ is a minimal subset and $x_{0} x_{1} \ldots x_{n+1}, x_{i} \in X^{ \pm 1}$, is a sink with no subword equal to the identity, then by applying the arguments above we get that there is a subword $x_{i} \ldots x_{j}$ such that $x_{i}, \ldots, x_{j}$ all lie in the same conjugate of the base $B$ and $x_{i} \ldots x_{j}$ is conjugate to an element of $K_{1}$ or $K_{-1}$. Thus we have the main result of Peczynski and Reiwer [5].

\section{REFERENCES}

1. A. H. M. Hoare, On length functions and Nielsen methods in free groups, J. London Math. Soc. (2) 14 (1976), 188-192.

2. R. C. Lyndon, Length functions in groups, Math. Scand. 12 (1963), 209-234.

3. H. Neumann, Generalized free products with amalgamated subgroups, Amer. J. Math. 70 (1948), 590-625.

4. W. Magnus, A. Karrass, and D. Solitar, Combinatorial Group Theory: Presentations of Groups in Terms of Generators and Relations (Pure and Applied Mathematics 13), Interscience Publishers - John Wiley and Sons, New York - London - Sydney, 1966.

5. N. Peczynski and W. Reiwer, On cancellations in HNN-Groups, Math. Z. 158 (1978), 79-86.

6. H. Zieschang, Über die Nielsensche Kürzungsmethode in freien Produkten mit Amalgam, Invent. Math. 10 (1970), 4-37.

THE UNIVERSITY OF BIRMINGHAM DEPARTMENT OF PURE MATHEMATICS BIRMINGHAM B15 2TT ENGLAND 